\title{
Tratamiento protésico y quirúrgico del maxilar atrófico.
}

\section{Prosthetic and surgical treatment of the atrophic maxilla.}

\author{
Yuri Cataldo ${ }^{1 *}$, Cristian Fernández ${ }^{2}$
}

1. Hospital Clínico Dra. Eloísa Díaz, Rehabilitación

Oral, Servicio Dental, Santiago, Chile.

2. Universidad de Chile: Área de Prótesis,

Departamento de rehabilitación oral, Facultad de

Odontología, Santiago, Chile.

* Correspondencia Autor: Yuri Cataldo. I Rehabilitación Oral, Servicio Dental, Hospital

Clínico Dra. Eloísa, Santiago, Chile: | Dirección:

Froilán Roa 6542, La Florida, Santiago,

Chile. | Teléfono: +56 96120 0966. | E-mail:

dr.yuricataldo@gmail.com

Trabajo recibido el 02/03/2020.

Aprobado para su publicación el 11/07/2020

\begin{abstract}
RESUMEN
Proponer un plan de tratamiento con técnicas quirúrgicas y protocolos protésicos predecibles representa un gran desafío profesional, especialmente en pacientes con un maxilar atrófico. Entre las alternativas terapéuticas, la prótesis híbrida sobre cuatro implantes dentales correctamente distribuidos permite formar un área de distribución de carga, la cual guarda relación con las necesidades biomecánicas del elemento protésico.
\end{abstract}

PALABRAS CLAVE:

Elevación piso sinusal; Maxilar atrófico; Prótesis híbrida.

Int. J. Inter. Dent Vol. 13(3); 165-167, 2020.

\section{ABSTRACT}

Proposing a treatment plan with surgical techniques and predictable prosthetic protocols means a great professional challenge, especially in patients with an atrophic maxilla. Among the therapeutic alternatives, the hybrid prosthesis on four correctly distributed dental implants, allows to form a load distribution area, which is related to the biomechanical needs of the prosthetic element.

\section{KEY WORDS:}

Sinus lift; Atrophic maxilla; Hybrid prosthesis.

Int. J. Inter. Dent Vol. 13(3); 165-167, 2020.

\section{INTRODUCCIÓN}

Actualmente, el tratamiento de pacientes totalmente desdentados a través de prótesis fijas de arco completo implanto asistidas, es un procedimiento rutinario y con sustento en la evidencia, con tasas de éxito y sobrevida reportadas sobre el $90 \%{ }^{(1-4)}$. En pacientes con maxilares atrófico, donde es complejo obtener una correcta distribución implantaria, pues no hay suficiente volumen de tejido óseo en el sector anterior y posterior para la inserción de implantes, se ha sugerido la utilización de técnicas quirúrgicas complementarias, como la elevación del piso sinusal, para resolver la falta de altura ósea. Este procedimiento ha demostrado resultados predecibles a largo plazo ${ }^{(5-8)}$.

\section{INFORME DEL CASO}

Paciente de sexo femenino de 58 años de edad, sin antecedentes de patologías sistémicas, desdentada total maxilar y parcial mandibular. Motivo de consulta funcional y estético, describe dificultad para comer y reparos estéticos de su prótesis removible. Al examen clínico se observan gran reabsorción del reborde residual, tejido blando con bajo nivel de encía adherida y soporte óseo disminuido (Figura 1).

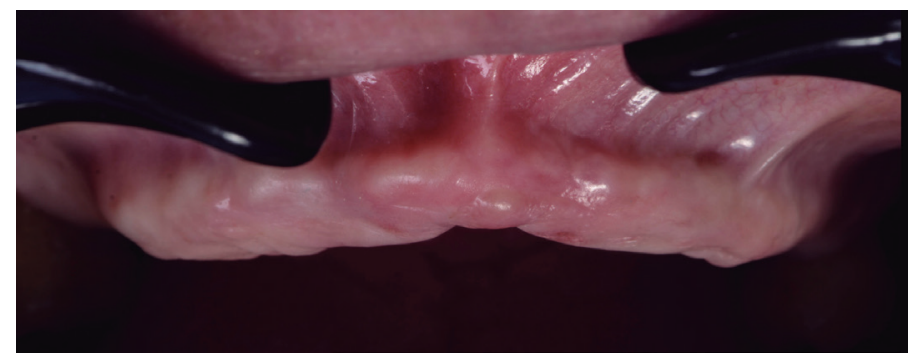

Figura 1. Visión frontal del maxilar con escasa altura, inserción alta de frenillos y poca cantidad de encía adherida.
Se le entregó a la paciente las alternativas de tratamiento que incluía una nueva prótesis removible mucosoportada o una prótesis híbrida fija sobre implantes, y eligió la prótesis implanto asistida, pues ya tuvo mala experiencia con la aparatología removible por falta de retención.

Para una correcta distribución implantaria que proporcione soporte, retención y estabilidad al elemento protésico en una rehabilitación de arco completo maxilar, la evidencia actual describe como protocolo considerar la inserción mínima de cuatro implantes dentales ${ }^{(9,10)}$ (Figura 2). Para este caso se seleccionaron implantes con un macrodiseño de cuerpo cónico de 1.5 grados (TS III SA Fixture Osstem ${ }^{\circledR}$ ), con los objetivos de aumentar el torque de inserción quirúrgico, y lograr estabilidad mecánica a largo plazo, a su vez el microdiseño con una superficie tratada por ácido incrementa el área de superficie, gracias a la formación de agujeros, muescas y protrusiones, que inducen a las plaquetas a secretar mediadores biológicos que estimulan la diferenciación osteoblástica, y promueven la adhesión y estabilización del coágulo sanguíneo, incluyendo la formación de matriz de fibrina, que actúa como un andamio osteoconductor para la migración de células osteogénicas, que conducen a la formación de hueso en la superficie del implante(11), es decir, aumenta la cantidad de contacto directo hueso-implante (BIC), este factor es fundamental para un caso de maxilar atrófico en donde el tejido óseo nativo es poco y se necesita lograr la mayor cantidad de tejido óseo circundante al implante.

Se realizó la inserción quirúrgica de 4 implantes (Figura 2):

- Implante 1 de $4.0 \mathrm{~mm}$. de diámetro por $10 \mathrm{~mm}$. de largo.

- Implante 2 de $4.0 \mathrm{~mm}$. de diámetro por $8.5 \mathrm{~mm}$. de largo.

- Implante 3 de $3.5 \mathrm{~mm}$. de diámetro por $10 \mathrm{~mm}$. de largo.

- Implante 4 de $4 \mathrm{~mm}$. de diámetro por $10 \mathrm{~mm}$. de largo.

Simultáneamente, se ejecutó una cirugía de elevación de seno maxilar bilateral (Figura 3), pues el hueso nativo antral en la zona del implante 1 , tenía una altura de $6.5 \mathrm{~mm}$ y en la zona del implante 4 , tenía una altura de $6 \mathrm{~mm}$. La técnica quirúrgica que se utilizó fue la de ventana lateral (LAS Kit - Osstem $^{\circledR}$ ), y se rellenó en ambos senos maxilares con 0,75 cc de injerto aloplástico (Q-Oss ${ }^{\circledR}-$ Osstem $^{\circledR}$ ) con partículas de tamaño $0.5 \sim 1.0 \mathrm{~mm}$, y un contenido de $20 \%$ hidroxiapatita (HA) y $80 \%$ fosfato tricálcico ( $\beta$-TCP). En la zona del defecto óseo generado por el 


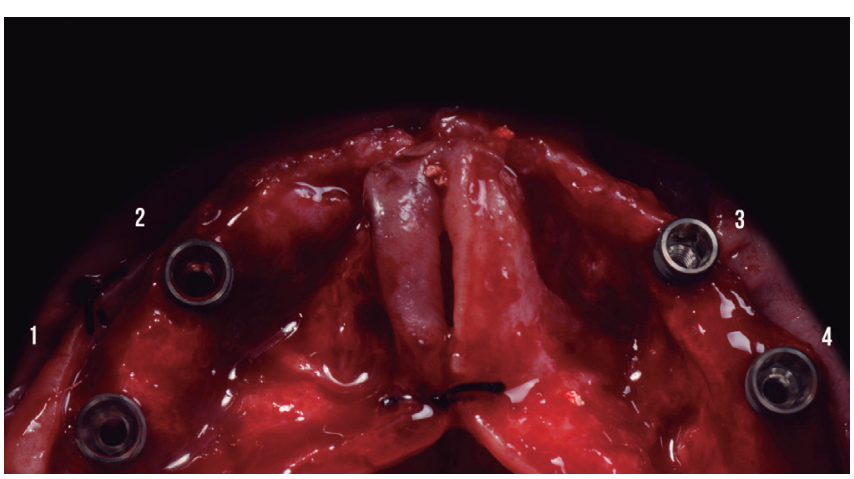

Figura 2. Distribución de 4 implantes en maxilar atrófico desdentado.

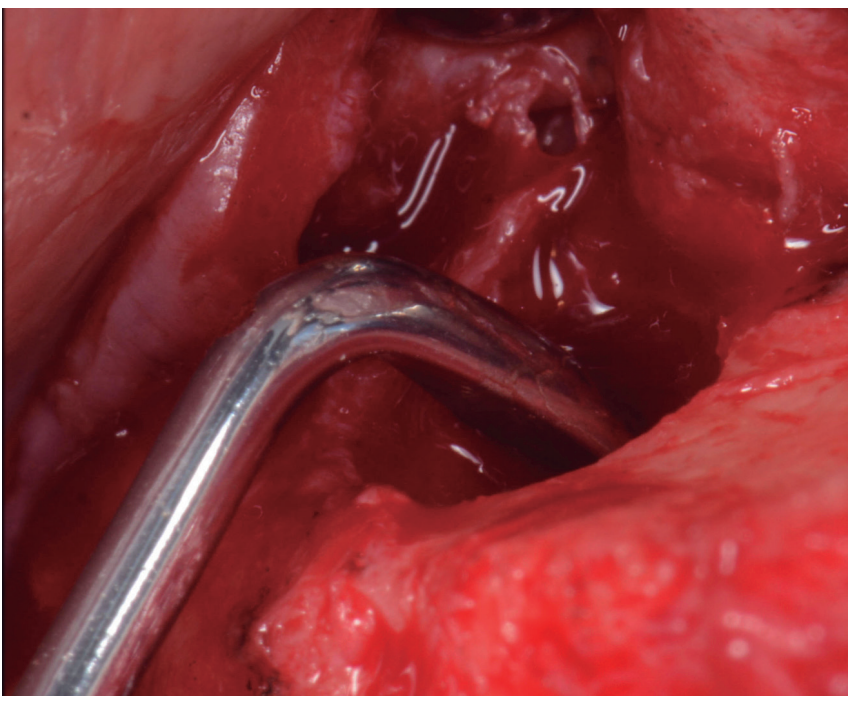

Figura 3. Osteotomía en pared lateral del seno maxilar y desprendimiento de la membrana de Scheneider con curetas.

acceso al seno maxilar, se utilizaron membranas biológicas de fibrina rica en plaquetas ${ }^{(12)}$, que se obtuvieron siguiendo el protocolo de Choukroun centrifugando a 3.000 revoluciones por minuto y por 10 minutos $^{(12,13)}$. Durante la inserción de los implantes hubo zonas en que se produjeron fenestraciones, y sobre estas, se realizó una regeneración ósea guiada con el mismo injerto $(0,5 \mathrm{cc})$ y membranas ${ }^{(12)}$.

Por la calidad del tejido óseo tipo IV, en la clasificación de Lekholm y Zarb ${ }^{(14)}$, y las cirugías complementarias realizadas, se consideró un protocolo de carga convencional(15), luego de 8 semanas desde la instalación de los implantes. Posteriormente, se realizó la cirugía de conexión, selección de pilares protésicos Multi-Unit rectos $(2 \mathrm{~mm}$. de altura gingival), y un flujo convencional de trabajo para realizar una barra de cobalto-cromo sobre pilares calcinables con base cromo-cobalto (Figura 4).

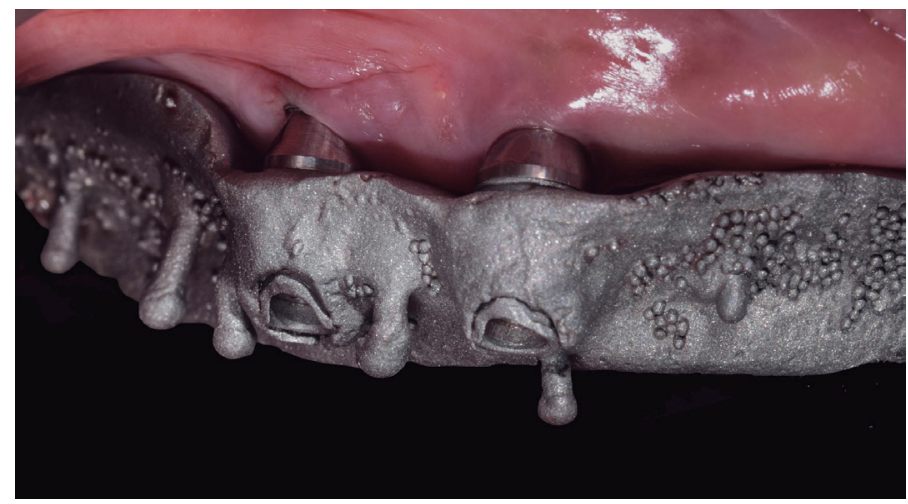

Figura 4. Prueba clínica de ajuste pasivo, se observa el íntimo ajuste entre la estructura y los pilares protésicos.
Se diseñó una prótesis híbrida metal acrílica implanto asistida (Figura 5) para el maxilar y una prótesis parcial removible metal acrílica para la mandíbula, con las cuales se restituyó función y estética orofacial (Figura 6 ). Finalmente, se indicó una radiografía panorámica de control a los 3 meses posterior a la instalación de los elementos protésicos (Figura 7).

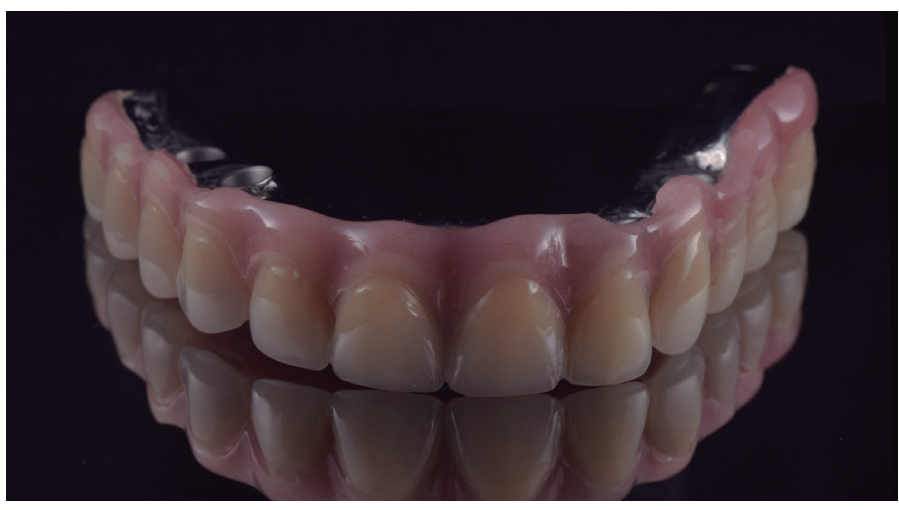

Figura 5. Prótesis híbrida terminada.

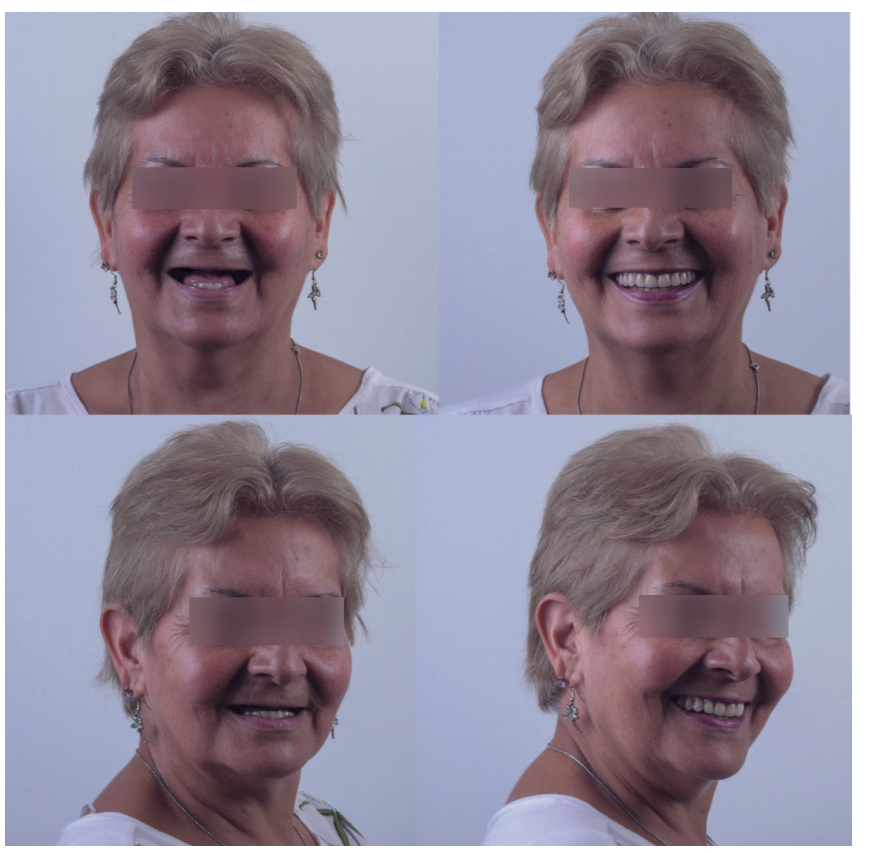

Figura 6. Resultado estético logrado.

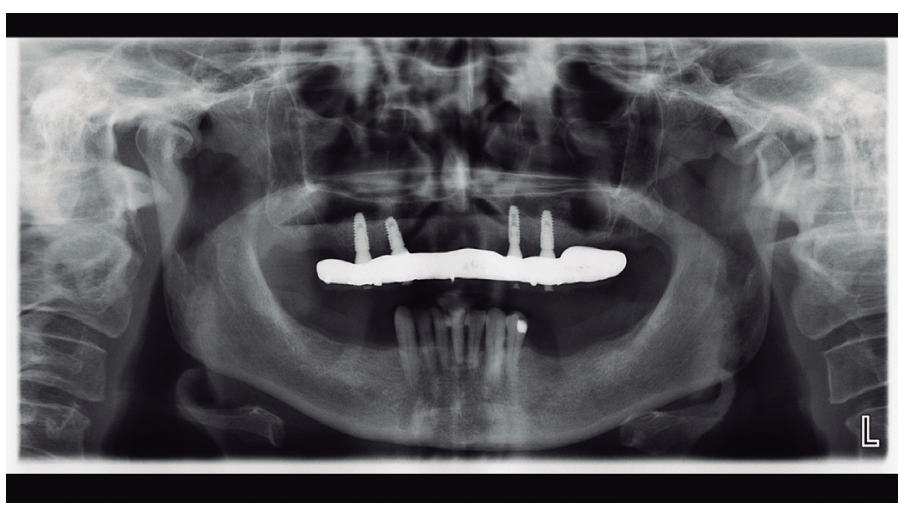

Figura 7. Ortopantomografía de control tras la instalación del elemento protésico.

\section{DISCUSIÓN Y CONCLUSIÓN}

Entre las alternativas terapéuticas para el paciente desdentado completo, las prótesis implanto asistidas representan, actualmente, la primera opción terapéutica. Este tratamiento, de acuerdo con la evidencia 
actual, permite la obtención de resultados armónicos y funcionales ${ }^{(1-3)}$.

Siguiendo los postulados y recomendaciones de diversos estudios y guías clínicas disponibles ${ }^{(1-4)}$, se decidió realizar un protocolo de carga protésica convencional que corresponde a la instalación del aparato protésico, luego de al menos 2 meses de reparación ósea desde la inserción de los implantes ${ }^{(16)}$. Las variables evaluadas para esta decisión contemplan principalmente la realización de cirugías complementarias, en este caso, la elevación del piso sinusal y regeneración ósea guiada horizontal. Dichos procedimientos implican la espera de los tiempos necesarios para los procesos fisiológicos de regeneración del tejido óseo(17).

La elevación del seno maxilar mediante la técnica de ventana lateral es un procedimiento que ha demostrado, en diversas publicaciones ${ }^{(5,6)}$, ser predecible en el aumento óseo de la región posterior maxilar, junto con el uso de material de injerto y membranas de fibrina rica en plaquetas. En este caso, dicho procedimiento permitió posicionar implantes en la zona de molares, logrando una distribución implantaria que asegura una correcta biomecánica del elemento protésico.

Una alternativa terapéutica a la cirugía complementaria del seno maxilar, es la instalación de implantes inclinados, sorteando la neumatización del seno maxilar o la reabsorción severa de la mandíbula, planteado por Krekmanov y Malo(18). Esta técnica permite instalar implantes de mayor longitud, así como también, mejorar la distribución poligonal de soporte protésico y reducir el número de implantes, sin la necesidad de realizar un injerto óseo de relleno de seno maxilar. Para considerar esta alternativa es fundamental la evaluación de la extensión y morfología, de la zona anterior del seno maxilar, que permita ejecutar adecuadamente este protocolo(18,19)

Otra opción para el tratamiento de maxilares atróficos es la utilización de anclajes remotos, como los implantes a los huesos cigomáticos y pterigoides, que presentan altas tasas de sobrevida del $95.2 \%$ y $94.7 \%$ respectivamente ${ }^{(20,21)}$. Sin embargo, requieren de un cirujano con gran habilidad y experiencia para su ejecución, puesto que se relacionan a zonas anatómicas extremadamente sensibles, y sus complicaciones son mas difíciles de manejar al compararlas con las que podemos tener en una elevación de seno maxilar o regeneración ósea guiada horizontal(21).

Los implantes cigomáticos presentan una ventaja comparativa al protocolo presentado, pues permiten realizar carga inmediata de los implantes ${ }^{(15)}$ (instalación de la prótesis hasta 1 semana desde la inserción de los implantes) y evitar las cirugías reconstructivas ${ }^{(21)}$. Las alternativas quirúrgicas en un protocolo de implantes inclinados e implantes al hueso pterigoides o cigomáticos, no presentan para este caso clínico ventajas comparativas, ya que la neumatización del seno maxilar era extensa, por tanto, no se hubiese logrado una distribución de implantes en la zona posterior maxilar y el costo asociado a esas técnicas es más elevado ${ }^{(3)}$.

La prótesis de arco completo sobre 4 implantes dentales es un tratamiento con tasas de éxito sobre el $90 \%^{(1,2)}$ y es el resultado de la optimización de: función, biomecánica, asequibilidad y comodidad del paciente ${ }^{(3)}$. Los factores asociados con la complejidad del tratamiento y la comodidad del paciente, como la calidad de la prótesis, el número de cirugías y la duración del tiempo de tratamiento, favorecen significativamente el concepto de tratamiento sobre 4 implantes dentales en comparación con las modalidades de tratamiento convencionales con 6 implantes ${ }^{(3,9,10)}$. Sin embargo, este enfoque sigue siendo controvertido ya que la pérdida de un implante conduce al fracaso de la prótesis, con un compromiso significativo del resultado ${ }^{(10)}$.

Desde el punto de vista biopsicocial, la rehabilitación implanto asistida fija sobre implantes, está enfocada a mejorar la percepción de calidad de vida en los pacientes adultos mayores, que generalmente son personas carenciadas. Es por esto, que seleccionar técnicas quirúrgicas ${ }^{(1,2,5,6)}$ y protocolos protésicos predecibles ${ }^{(15)}$ permite resolver, con resultados satisfactorios, la gran mayoría de los casos clínicos de los pacientes desdentados completos.

\section{RELEVANCIA CLÍNICA}

El desdentamiento tiene una alta prevalencia en Chile, pues del grupo de adultos entre $35-44$ años, solo un $20 \%$ conserva todos sus dientes ${ }^{(22)}$ Actualmente, el uso de implantes dentales guiados protésicamente es el tratamiento a elección para los desdentados totales pues tiene poca morbilidad y altas tasas de éxito y sobrevida. El motivo de este reporte clínico es poder describir técnicas quirúrgicas y protésicas para abordar casos de maxilares atróficos y analizar el resultado obtenido en base a la evidencia científica actual.

\section{CONFLICTO DE INTERESES}

Autores no declaran conflictos de interés.

\section{Bibliografía}

1. Jemt T, Johansson J. Implant treatment in the edentulous maxillae: A 15-year follow-up study on 76 consecutive patients provided with fixed prostheses. Clin Implant Dent Relat Res. 2006;8(2):61-9.

2. Maló $P$, Rangert B, Nobre M. All-on-4 immediate-function concept with Brånemark System $®$ implants for completely edentulous maxillae : a 1-year retrospective clinical study. Clin Implant Dent Relat Res. 2005;7(Suppl 1):S88-94.

3. Babbush CA, Kanawati A, Kotsakis GA, Hinrichs JE. Patient-related and financia outcomes analysis of conventional full-arch rehabilitation versus the all-on-4 concept: A cohort study. Implant Dent. 2014;23(2):218-24.

4. Balshi TJ, Wolfinger GJ, Slauch RW, Balshi SF. A Retrospective analysis of 800 Brånemark System implants following the all-on-fourTM protocol. J Prosthodont. 2014;23(2):83-8

5. Pjetursson BE, Tan WC, Zwahlen M, Lang NP. A systematic review of the success of sinus floor elevation and survival of implants inserted in combination with sinus floor elevation: Part I: Lateral approach. J Clin Periodontol. 2008;35(Suppl. 8):216-40. 6. Raghoebar GM, Onclin P, Boven GC, Vissink A, Meijer HJA. Long-term effectiveness of maxillary sinus floor augmentation: A systematic review and metaanalysis. J Clin Periodontol. 2019;46(S21):307-18.

7. Starch-Jensen T, Aludden H, Hallman M, Dahlin C, Christensen AE, Mordenfeld A. A systematic review and meta-analysis of long-term studies (five or more years) assessing maxillary sinus floor augmentation. Int J Oral Maxillofac Surg. 2018;47(1):103-16.

8. Corbella S, Taschieri S, Del Fabbro M. Long-term outcomes for the treatment of atrophic posterior maxilla: A systematic review of literature. Clin Implant Dent Relat Res. 2015;17(1):120-32.

9. de Luna Gomes JM, Lemos CAA, Santiago Junior JF, de Moraes SLD, Goiato MC, Pellizzer EP. Optimal number of implants for complete-arch implant-supported prostheses with a follow-up of at least 5 years: A systematic review and metaanalysis. J Prosthet Dent. 2019;121(5):766-774.e3.

10. Daudt Polido W, Aghaloo T, Emmett TW, Taylor TD, Morton D. Number of implants placed for complete-arch fixed prostheses: A systematic review and metaanalysis. Clin Oral Implants Res. 2018;29(May):154-83.

11. Almas K, Smith S, Kutkut A. What is the best micro and macro dental implant topography? Dent Clin North Am. 2019;63(3):447-60.
12. Choukroun J, Diss A, Simonpieri A, Girard MO, Schoeffler C, Dohan SL, et al. Platelet-rich fibrin (PRF): A second-generation platelet concentrate. Part IV: Clinical effects on tissue healing. Oral Surg Oral Med Oral Pathol Oral Radiol Endod. 2006;101(3):56-60

13. Borie E, Oliví DG, Orsi IA, Garlet K, Weber B, Beltrán V, et al. Platelet-rich fibrin application in dentistry: A literature review. Int J Clin Exp Med. 2015;8(5):7922-9.

14. Lekholm U, Zarb G. Patient selection and preparation. In: Branemark P, Zarb $\mathrm{G}$, Albrektsson T, editors. Tissue integrated prostheses: osseointegration in clinical dentistry. Quintessence Publishing Company; 1985. 199-209 p.

15. Wismeijer D, Buser D, Belser U. ITI Treatment Guide, Vol 4: Loading Protocols in Implant Dentistry: Edentulous Patients. Quintessence Publishing Company; 2010. $248 \mathrm{p}$.

16. Esposito M, Grusovin MG, Willings M, Coulthard P, Worthington HV. The effectiveness of immediate, early, and conventional loading of dental implants: a Cochrane systematic review of randomized controlled clinical trials. Int J Oral Maxillofac Implant. 2007;22(6):893-904.

17. Chen S, Buser D, Wismeijer D. ITI Treatment Guide, Volume 5-Sinus Floor Elevation Procedures. Chen S, Buser D, Wismeijer D, editors. Quintessence Publishing Company; 2011. $232 \mathrm{p}$

18. Krekmanov L, Kahn M, Rangert B, Lindström H. Tilting of posterior mandibular and maxillary implants for improved prosthesis support. Int J Oral Maxillofac Implants. 2000;15(3):405-14

19. Aparicio C, Perales P, Rangert B. Tilted implants as an alternative to maxillary sinus grafting: A clinical, radiologic, and periotest study. Clin Implant Dent Relat Res. 2001;3(1):39-49.

20. Valerón JF, Valerón PF. Long-term results in placement of screw-type implants in the pterygomaxillary-pyramidal region. Int J Oral Maxillofac Implants. 2007;22(2):195-200

21. Chrcanovic BR, Albrektsson T, Wennerberg A. Survival and complications of zygomatic implants: an updated systematic review. J Oral Maxillofac Surg 2016;74(10):1949-64. 